\title{
Dinâmica da distribuição diamétrica de povoamentos de Pinus taeda, na região de Caçador, SC
}

\author{
Saulo Jorge Téo1, Samara Pozzan da Rocha1 ${ }^{1}$, Aline Cristina Bortoncello ${ }^{1}$, Rafaela Antunes Paz ${ }^{1}$, Reinaldo Hoinacki da Costa²
}

${ }^{1}$ Universidade do Oeste de Santa Catarina (UNOESC), Rua Dirceu Giordani, CEP 89820-000, Xanxerê, SC, Brasil

2Juliana Florestal, Rua Padre Feijó, CEP 89500-000, Caçador, SC, Brasil

"Autor correspondente:

sauloteo@yahoo.com.br

Termos para indexação:

Estrutura horizontal

Função de densidade probabilística

Teste de aderência de Kolmogorov-Smirnov

Index terms:

Horizontal structure

Probability density function

Kolmogorov-Smirnov adherence test

Histórico do artigo:

Recebido em 28 dez 2010

Aprovado em 17 ago 2011

Publicado em 30 set 2011

doi: $10.4336 / 2011 . p f b .31 .67 .183$
Resumo - O objetivo deste trabalho foi ajustar as funções de densidade probabilísticas (fdp's) Normal e $\mathrm{S}_{\mathrm{B}}$ de Johnson, para descrever as mudanças, com a idade, na estrutura diamétrica de povoamentos de Pinus taeda L., na região de Caçador, SC. Os dados utilizados foram provenientes de parcelas temporárias e permanentes, com idades variando de 5 a 11,9 anos, agrupadas em classes com intervalos de 1 ano. Foram ajustadas as fdp's e obtidas as estimativas dos parâmetros de assimetria e curtose. Por meio do teste de Kolmogorov-Smirnov, verificou-se que, para as idades de 5 a 7,9 anos, as fdp's Normal e $\mathrm{S}_{\mathrm{B}}$ de Johnson não apresentaram aderência satisfatória. As fdp's $S_{B}$ de Johnson e Normal apresentaram melhor aderência para as idades de 8 a 9,9 e 10 a 11,9 anos, respectivamente. Utilizando a fdp Normal para analisar a dinâmica da distribuição diamétrica, verificou-se um deslocamento das curvas de distribuição diamétrica para a direita, um "achatamento" e um "alargamento" destas curvas ao longo dos anos. A assimetria foi negativa para todas as idades, exceto para as idades de 8 a 8,9 anos. A curtose foi leptocúrtica para todas as idades, e platicúrtica para as idades de 8 a 8,9 anos.

\section{Dynamics of the diameter distribution of Pinus taeda stands, in the region of Caçador, SC, Brazil}

\begin{abstract}
The objective of this work was to fit the probability density functions Normal and $\mathrm{S}_{\mathrm{B}}$, to describe the changes, with age, in the diameter distribution of loblolly pine (Pinus taeda) stands, in the region of Caçador, Santa Catarina State, Brazil. The data came from permanent and temporary plots, with ages ranging from 5 to 11,9 , grouped in age classes with intervals of 1 year. The probability density functions were fitted and, the Kolmogorov-Smirnov test and the parameters of skewness and kurtosis were calculated. Basing on Kolmogorov-Smirnov test it was verified that, for ages from 5 to 7.9 years old, the Normal and $\mathrm{S}_{\mathrm{B}}$ functions didn't show satisfactory adherence. The $\mathrm{S}_{\mathrm{B}}$ and Normal functions showed better adherence to the ages from 8 to 9.9 and from 10 to 11.9 years old, respectively. Using the Normal probability density functions to analyze the dynamics of the diameter distribution, it was found that there was a shift of the diameter distribution curves to the right, a "flatness" and an "wideness" of these curves, over the years. The skewness was negative for all ages, except for the age from 8 to 8.9 years old. The kurtosis was leptokurtic for all ages, except for age from 8 to 8.9 years old, which was platykurtic.
\end{abstract}




\section{Introdução}

Para otimizar a produção florestal e atender às exigências e necessidades do mercado consumidor, é importante ter um bom conhecimento e planejamento das florestas por meio do controle de sua densidade. Para tanto, pode ser utilizada a distribuição diamétrica, que permite estimar o número de árvores por hectare existentes em cada classe de diâmetro.

Conforme Schaaf et al. (2006), a maneira mais adequada para entender o desenvolvimento da distribuição dos diâmetros de uma floresta é monitorando-a sistematicamente e por longos períodos, sendo que a instalação de parcelas permanentes é a metodologia mais adequada para tal finalidade. Para Machado et al. (2009a), ao realizar sucessivas medições da floresta, se obtém uma poderosa ferramenta na avaliação da dinâmica de florestas nativas e plantadas.

De acordo com Scolforo (2006), em florestas plantadas a distribuição diamétrica é importante para programar a predição ou prognose da produção florestal. Em florestas nativas que não tem idade definida, ela permite obter a amplitude dos diâmetros e a densidade de indivíduos por classe diamétrica.

A distribuição diamétrica é o meio mais eficaz e simples para descrever a estrutura de um povoamento, a qual consiste na distribuição de espécies e dimensões das árvores em relação a uma unidade de área, sendo resultado das condições ambientais, do hábito de crescimento e das práticas de manejo das espécies (Loetsch et al., 1973; Clutter et al., 1983).

O conhecimento da estrutura diamétrica permite avaliar o comportamento da espécie, considerando os vários fatores que podem interferir em seu crescimento, favorecendo o manejo da mesma, considerando que todas as atividades e manejo de exploração comercial causam alterações na estrutura diamétrica (Bartoszeck et al., 2004).

A distribuição dos diâmetros tem importante aplicação no manejo florestal. Scolforo (2006) ressaltou que, em nível operacional, a distribuição diamétrica possibilita que seja implementada técnica para remover árvores por classes de diâmetro, desde que seja utilizado o conceito de floresta balanceada descrito por De Liocourt (1898) e Meyer (1952). Além disso, Scolforo (1998) complementou que a análise da distribuição dos diâmetros possibilita caracterizar e indicar o estoque de madeira disponível antes de realizar uma exploração, bem como fornece informações sobre a necessidade de reposição florestal.

A distribuição diamétrica também é aplicada no sortimento florestal (Schneider et al., 2008) e em modelos de produção por classe diamétrica, também denominados de modelos implícitos de crescimento e produção (Scolforo, 2006).

A distribuição diamétrica pode ser unimodal, multimodal e decrescente (Loetsch et al., 1973) e pode ser representada por uma tabela, histograma de frequências ou por funções de densidade probabilística (fdp's).

Atualmente, conforme Machado et al. (2009a), a melhor forma de descrever a estrutura diamétrica de uma espécie ou de uma floresta é por meio das funções de densidade probabilística (fdp's). As fdp's possibilitam obter a probabilidade de as árvores ocorrerem dentro de intervalos ou classes de diâmetro, em que haja um limite inferior e um superior. Algumas funções de densidade probabilísticas são: Weibull, Meyer, Gamma, $\mathrm{S}_{\mathrm{B}}$ de Johnson, Normal, Log-normal, Beta (Carelli Netto, 2008), "A" de Charlier, Peaerl-Reed (Alvarez Gonzalez \& Ruiz Gonzalez, 1998), de Weber (Machado et al., 2009a; Machado et al., 2010), de Quadros e de Pélico Netto (Machado et al., 2010).

Para avaliar a qualidade do ajuste das funções de densidade probabilísticas são realizados testes de aderência. Conforme Carelli Netto (2008), alguns desses testes são: teste de Kolmogorov-Smirnov (KS), de Anderson-Darling (AD), Qui-quadrado $\left(\chi^{2}\right)$, ShapiroWilk (W) e teste de Cramér-von Mises (W-Sq).

A evolução das curvas de distribuição é descrita pelos parâmetros de assimetria e curtose. A assimetria refere-se ao afastamento da simetria de uma distribuição de frequência em relação à curva Normal, e a curtose é definida como o grau de "achatamento" de uma distribuição em relação à curva Normal (Machado et al., 2006).

Devido à sua importância, a distribuição dos diâmetros tem sido estudada para diferentes povoamentos, espaçamentos e densidades e, para descrevê-las, diversas funções de densidade probabilísticas têm sido ajustadas. Dentre esses estudos, merecem destaque os de Alvarez Gonzalez e Ruiz Gonzalez (1998), Bartoszeck et al. (2004), Machado et al. (2006), Nogueira et al. (2006), Carelli Netto (2008), Schneider et al. (2008), Machado et al. (2009a), Machado et al. (2009b), Machado et al. (2010), entre outros. 
Este estudo teve como objetivo ajustar as funções de densidade probabilística Normal e $\mathrm{S}_{\mathrm{B}}$ de Johnson para descrever a estrutura diamétrica de povoamentos de Pinus taeda, com idades entre 5 e 11,9 anos, localizados na região de Caçador, $\mathrm{SC}$.

\section{Material e métodos}

Os dados para a realização da presente pesquisa foram obtidos de parcelas permanentes e temporárias, de povoamentos de Pinus taeda, da empresa Juliana Florestal Ltda., localizados na região de Caçador, SC.

Segundo a Empresa de Pesquisa Agropecuária e Extensão Rural de Santa Catarina (EPAGRI, 2010), o Município de Caçador apresenta o clima Cfb da classificação climática de Köeppen, o qual corresponde ao clima temperado, constantemente úmido, sem estação seca e com verão fresco. A temperatura média anual deste município varia de $14,4{ }^{\circ} \mathrm{C}$ a $16,3{ }^{\circ} \mathrm{C}$, sendo que a temperatura média dos meses mais quentes varia de $20,7^{\circ} \mathrm{C}$ a $23,7{ }^{\circ} \mathrm{C}$, e dos meses mais frios de $9,1^{\circ} \mathrm{C}$ a $10,8^{\circ} \mathrm{C}$. A precipitação pluviométrica total anual é de $1.490 \mathrm{~mm}$ a $2.100 \mathrm{~mm}$ e a umidade relativa do ar é, em média, $78,1 \%$ a $82,9 \%$.

$\mathrm{Na}$ região de Caçador ocorriam, originalmente, a Floresta Ombrófila Mista e Campos do Planalto (EPAGRI, 2010). Segundo o Centro de Informações de Recursos Ambientais e de Hidrometeorologia de Santa Catarina (CIRAM, 2002), as classes predominantes de solos da região dos povoamentos em estudo são Nitossolos, Neossolos e Cambissolos.

As parcelas permanentes e temporárias variaram de $300 \mathrm{~m}^{2}$ a $900 \mathrm{~m}^{2}$ e foram distribuídas de maneira aleatória. Por meio de inventários florestais contínuos, realizados de 2007 a 2009, foram feitas medições das circunferências à altura do peito e das alturas totais das árvores, com o uso de fita métrica e hipsômetro, respectivamente. Os povoamentos possuíam idade de 5 a 11,9 anos e espaçamento de 2,5 $\mathrm{m} \mathrm{x} \mathrm{2,0} \mathrm{m.} \mathrm{No}$ total, foram medidas 891 parcelas, entre temporárias e permanentes, e o número total de árvores medidas foi de 77.403 árvores.

Para o processamento dos dados, as parcelas foram agrupadas em classes de idades, como segue: de 5 a 5,9 anos, de 6 a 6,9 anos, de 7 a 7,9 anos, de 8 a 8,9 anos, de 9 a 9,9 anos, de 10 a 10,9 anos e de 11 a 11,9 anos.

O processamento dos dados se constituiu no agrupamento dos dados de diâmetro em diferentes classes diamétricas, no ajuste das funções de densidade probabilística (fdp) Normal e $\mathrm{S}_{\mathrm{B}}$ de Johnson, na realização do teste de aderência de KolmogorovSmirnov e no cálculo dos parâmetros de assimetria e curtose.

Para agrupar as árvores em classes de diâmetro foi escolhida, arbitrariamente, uma amplitude de $3 \mathrm{~cm}$, pois, de acordo com Schaaf et al. (2006), não há regra definida quanto à amplitude das classes.

Em seguida, para descrever a estrutura diamétrica dos povoamentos de Pinus taeda, da região de Caçador, SC, foram ajustadas as funções de densidade probabilística Normal e $\mathrm{S}_{\mathrm{B}}$ de Johnson.

A função de densidade probabilística Normal é descrita pela seguinte expressão matemática:

$$
f(x)=\frac{1}{\sigma \sqrt{2 \pi}} \cdot e^{\left\{-\frac{1}{2}\left[\frac{(x-d)^{2}}{\sigma^{2}}\right]\right\}}
$$

onde:

$x=$ variável independente, neste caso, o diâmetro;

$\sigma^{2}=$ variância dos diâmetros;

$\sigma=$ desvio padrão dos diâmetros;

$\bar{d}=$ média dos diâmetros.

A fdp $\mathrm{S}_{\text {B }}$ de Johnson foi ajustada com a seguinte expressão matemática:

$$
f(x)=\frac{\delta}{\sqrt{2 \pi}} \cdot \frac{\lambda}{(x-\varepsilon)(\lambda+\varepsilon-x)} \cdot e^{\left\{-\frac{1}{2}\left[\gamma+\delta \cdot \ln \left(\frac{x-\varepsilon}{\lambda+\varepsilon-x}\right)\right]^{2}\right\}}
$$

onde:

$x=$ definido anteriormente;

$\varepsilon=$ indica locação;

$\gamma=$ indica escala;

$\delta$ e $\gamma=$ determinam a forma da distribuição. Enquanto $\delta$ indica a curtose (mais achatada ou pontiaguda), $\gamma$ indica assimetria.

A fdp $\mathrm{S}_{\mathrm{B}}$ de Johnson foi ajustada e associada às porcentagens de $5 \%, 20 \%, 50 \%, 70 \%$ e $90 \%$ do diâmetro mínino $\left(\mathrm{d}_{\text {min. }}\right)$, sendo que para cada idade foi escolhida a fdp $\mathrm{S}_{\mathrm{B}}$ de Johnson que apresentou o menor valor de $D_{\text {calc }}$ do teste de Kolmogorov-Smirnov.

O teste de aderência de Kolmogorov-Smirnov foi aplicado para avaliar a qualidade do ajuste produzido pelas fdp's Normal e $\mathrm{S}_{\mathrm{B}}$ de Johnson.

Para a seleção da melhor fdp para descrever a dinâmica da distribuição diamétrica dos povoamentos, realizou- 
se um ranking, sendo que a fdp escolhida foi a que, na maioria das idades, teve o primeiro lugar neste ranking. $\mathrm{O}$ primeiro lugar foi atribuído à fdp que apresentou o menor valor de $D_{\text {calc }}$ de Kolmogorov-Smirnov.

Para descrever as formas e a evolução das curvas de distribuição, procedeu-se ao cálculo dos parâmetros de assimetria $\left(\gamma_{1}\right)$ e curtose $\left(\gamma_{2}\right)$.

$O$ parâmetro de assimetria $\left(\gamma_{1}\right)$ foi obtido por meio do quociente entre terceiro momento centrado na média $\left(\mathrm{m}^{3}\right)$ e o cubo do desvio padrão. A assimetria das distribuições diamétricas foi avaliada de acordo com o valor de $\gamma_{1}$, da seguinte maneira:

A distribuição é simétrica se: $\gamma_{1} \cong 0$;

A distribuição é assimétrica negativa ou à direita se: $\gamma_{1}<0$

A distribuição é assimétrica positiva ou à esquerda se: $\gamma_{1}>0$.

O parâmetro de curtose $\left(\gamma_{2}\right)$ é definido pelo quociente entre o quarto momento centrado na média $\left(\mathrm{m}^{4}\right)$ e o quadrado da variância, menos três. A curtose das distribuições diamétricas foi avaliada de acordo com o valor de $\gamma_{2}$, da seguinte maneira:

Se a curtose for em torno de zero $\left(\gamma_{2} \cong 0\right.$ ), a distribuição diamétrica é mesocúrtica;

Se a curtose for negativa $\left(\gamma_{2}<0\right)$, a distribuição diamétrica é platicúrtica;

Se a curtose for positiva $\left(\gamma_{2}>0\right)$, a distribuição diamétrica é leptocúrtica.

\section{Resultados e discussão}

\section{Distribuição diamétrica}

A Tabela 1 apresenta o número de árvores por hectare, o diâmetro mínimo, o diâmetro máximo, a amplitude dos diâmetros, a média, a variância e o desvio padrão dos diâmetros, por classe de idade, dos povoamentos de Pinus taeda da região de Caçador, SC, os quais são necessários para avaliar a distribuição diamétrica destes povoamentos.

Tabela 1. Caracterização da base de dados, por classe de idade, dos povoamentos de Pinus taeda L., da região de Caçador, SC.

\begin{tabular}{cccccccc}
\hline $\begin{array}{c}\text { Idade } \\
\text { (anos) }\end{array}$ & $\mathbf{N ~ h a}^{-1}$ & $\begin{array}{c}\text { Diâmetro } \\
\text { mínimo } \\
(\mathbf{c m})\end{array}$ & $\begin{array}{c}\text { Diâmetro } \\
\text { máximo } \\
\mathbf{( c m )}\end{array}$ & $\begin{array}{c}\text { Amplitude } \\
\text { de diâmetros } \\
(\mathbf{c m})\end{array}$ & $\begin{array}{c}\text { Média } \\
(\mathbf{c m})\end{array}$ & $\begin{array}{c}\text { Variância } \\
\left.\mathbf{( c m}^{\mathbf{}}\right)\end{array}$ & $\begin{array}{c}\text { Desvio padrão } \\
(\mathbf{c m})\end{array}$ \\
\hline $5-5,9$ & 1.570 & 3,18 & 24,51 & 21,33 & 13,89 & 6,219 & 2,494 \\
$6-6,9$ & 1.559 & 3,80 & 30,24 & 26,42 & 15,97 & 7,771 & 2,788 \\
$7-7,9$ & 1.616 & 4,14 & 29,92 & 25,78 & 17,54 & 10,322 & 3,213 \\
$8-8,9$ & 1.487 & 4,78 & 33,42 & 28,65 & 19,18 & 19,672 & 4,435 \\
$9-9,9$ & 1.358 & 5,41 & 25,78 & 20,37 & 17,85 & 10,774 & 3,282 \\
$10-10,9$ & 1.106 & 5,41 & 35,33 & 29,92 & 20,45 & 18,935 & 4,351 \\
$11-11,9$ & 891 & 5,41 & 37,24 & 31,83 & 22,71 & 31,258 & 5,591 \\
\hline
\end{tabular}

$\mathrm{O}$ número de árvores por hectare diminuiu com o avanço da idade, exceto da classe de idade de 6 a 6,9 anos para a classe de idade de 7 a 7,9 anos (Tabela 1). A redução no número de árvores por hectare da menor para a maior classe de idade, quando ocorreu, foi devido à mortalidade das árvores ao longo do tempo, bem como devido aos desbastes que ocorreram aos 9 anos.

Para o total de dados, o diâmetro variou de $3,18 \mathrm{~cm}$ a $37,24 \mathrm{~cm}$. Quando analisada para cada classe de idade, a amplitude dos valores de diâmetro não apresentou nenhuma tendência clara de aumento ou diminuição com a idade (Tabela 1).

A média dos diâmetros variou de $13,89 \mathrm{~cm}$ a 22,71 $\mathrm{cm}$ e aumentou da menor para a maior classe de idade, com exceção da classe de idade de 8 a 8,9 anos para a classe de idade de 9 a 9,9 anos (Tabela 1). O aumento da média aconteceu por causa do crescimento em diâmetro das árvores. Com o aumento da idade ocorreu uma diminuição do número de árvores por hectare $\mathrm{e}$ crescimento em diâmetro.

Um dos fatores responsáveis pela média dos diâmetros da classe de idade de 8 a 8,9 anos ser maior do que a média da classe de idade de 9 a 9,9 anos (Tabela 1) é a qualidade de sítio. A maior parte das parcelas com 8 a 8,9 anos encontra-se no sítio I, o qual possui melhores condições para o desenvolvimento e crescimento das árvores quando comparado com as condições de crescimento e desenvolvimento proporcionadas pelos 
sítios II e III. Além disso, a maior parte das parcelas da idade de 9 a 9,9 anos está localizada na classe de sítio III. A qualidade genética das árvores também pode ter influenciado para que a idade de 8 a 8,9 anos apresentasse maior média de diâmetros do que a idade de 9 a 9,9 anos.

Assim como a média, a variância e o desvio padrão também aumentaram com a idade, exceto da classe de idade de 8 a 8,9 anos para a classe de idade de 9 a 9,9 anos (Tabela 1). É possível que a variância e o desvio não tenham apresentado um padrão de aumento da classe de idade de 8 a 8,9 para 9 a 9,9 anos, devido ao efeito da qualidade de sítio sobre o desenvolvimento dos povoamentos.

O maior desvio padrão e a maior variância foram encontrados na maior classe de idade (Tabela 1), o que está de acordo com os resultados obtidos por Carelli Netto (2008), a qual estudou a distribuição diamétrica de Pinus taeda em diferentes idades e espaçamentos, no Município de Otacílio Costa, SC.

\section{Ajuste das funções de densidade probabilísticas}

Os parâmetros da função de densidade probabilística $\mathrm{S}_{\mathrm{B}}$ de Johnson, para as classes de idade de 5 a 11,9 anos, estão apresentados na Tabela 2. Para calcular a fdp Normal é necessário apenas a média $(\bar{d})$, a variância $\left(\sigma^{2}\right)$ e o desvio padrão $(\sigma)$ dos diâmetros, os quais podem ser observados na Tabela 1.

Tabela 2. Parâmetros da função de densidade probabilística $S_{B}$ de Johnson, ajustada por classe de idade, para os povoamentos de Pinus taeda L., da região de Caçador, SC.

\begin{tabular}{ccccc}
\hline $\begin{array}{c}\text { Idade } \\
\text { (anos) }\end{array}$ & $\varepsilon$ & $\lambda$ & $\sigma$ & $\gamma$ \\
\hline $5-5,9$ & 0,15915 & 24,50986 & 2,32104 & $-0,58928$ \\
$6-6,9$ & 0,19099 & 30,23944 & 2,61465 & $-0,23793$ \\
$7-7,9$ & 0,20690 & 29,92113 & 2,16497 & $-0,73020$ \\
$8-8,9$ & 4,29718 & 33,42254 & 1,73024 & 0,41169 \\
$9-9,9$ & 1,08225 & 25,78310 & 1,67161 & $-1,12705$ \\
$10-10,9$ & 0,27056 & 35,33240 & 1,86810 & $-0,57423$ \\
$11-11,9$ & 0,27056 & 37,24226 & 1,45176 & $-0,67450$ \\
\hline
\end{tabular}

$\varepsilon=$ locação; $\lambda=$ escala; $\sigma$ e $\gamma=$ forma da distribuição.

\section{Seleção das funções de densidade probabilísticas}

Por meio do teste de Kolmogorov-Smirnov, verificouse que, para as idades menores que 7,9 anos, as fdp's Normal e $S_{B}$ de Johnson não apresentaram aderência satisfatória com nível de significância de $5 \%$, pois o $D_{\text {calc }}$ foi maior do que o $D_{\text {tab. }}$. Portanto, para as idades menores que 7,9 anos, as fdp's Normal e $\mathrm{S}_{\mathrm{B}}$ de Johnson não representaram a distribuição diamétrica para o nível de significância de 5\% (Tabela 3).

Para as idades de 8 a 8,9 e 9 a 9,9 anos, a função que melhor representou a probabilidade da distribuição diamétrica foi a $\mathrm{S}_{\mathrm{B}}$ de Johnson, pois o $D_{\text {calc }}$ da fdp $\mathrm{S}_{\mathrm{B}}$ de Johnson foi menor do que o $D_{\text {calc }}$ da fdp Normal. Já para as idades de 10 a 10,9 e 11 a 11,9 anos, a função de densidade probabilística Normal foi a que melhor representou a probabilidade da distribuição diamétrica (Tabela 3).

Tabela 3. Resultados de $D_{\text {calc }}$ e $D_{\text {tab }}$ do teste de KolmogorovSmirnov, para as funções de distribuição probabilística Normal e $\mathrm{S}_{\mathrm{B}}$ de Johnson, ajustadas para povoamentos de Pinus taeda L., com idades de 5 a 11,9 anos, na região de Caçador, SC.

\begin{tabular}{|c|c|c|c|c|c|}
\hline \multirow{2}{*}{ 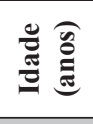 } & \multirow{2}{*}{$\begin{array}{c}\mathrm{N}^{0} \text { de } \\
\text { árvores }\end{array}$} & \multirow{2}{*}{ fdp } & \multicolumn{3}{|c|}{ Kolmogorov-Smirnov } \\
\hline & & & $D_{\text {calc }}$ & $D_{t a b}$ & Posição \\
\hline \multirow{2}{*}{$\begin{array}{l}\text { aे } \\
\text { nो } \\
\text { in }\end{array}$} & \multirow{2}{*}{22083} & Normal & $-0,01890$ & \multirow[b]{2}{*}{0,00966} & $1^{\circ}$ \\
\hline & & $\mathrm{S}_{\mathrm{B}}$ de Johnson & $-0,02313$ & & $2^{\circ}$ \\
\hline \multirow{2}{*}{$\begin{array}{l}a \\
\hat{b} \\
1 \\
b\end{array}$} & \multirow[b]{2}{*}{36849} & Normal & $-0,01386$ & \multirow{2}{*}{0,00708} & $1^{\circ}$ \\
\hline & & $\mathrm{S}_{\mathrm{B}}$ de Johnson & $-0,01509$ & & $2^{\circ}$ \\
\hline \multirow{2}{*}{$\frac{a}{2}$} & \multirow[b]{2}{*}{16094} & Normal & 0,03150 & \multirow[b]{2}{*}{0,01072} & $2^{\circ}$ \\
\hline & & $\mathrm{S}_{\mathrm{B}}$ de Johnson & 0,01254 & & $1^{\circ}$ \\
\hline \multirow{2}{*}{$\begin{array}{l}0 \\
\infty \\
1 \\
\infty\end{array}$} & \multirow[b]{2}{*}{806} & Normal & $-0,04131$ & \multirow[b]{2}{*}{0,04790} & $2^{\circ}$ \\
\hline & & $\mathrm{S}_{\mathrm{B}}$ de Johnson & $-0,02138$ & & $1^{\circ}$ \\
\hline \multirow{2}{*}{$\begin{array}{l}a \\
\text { aे } \\
a\end{array}$} & \multirow[b]{2}{*}{568} & Normal & 0,05243 & \multirow[b]{2}{*}{0,05706} & $2^{\circ}$ \\
\hline & & $\mathrm{S}_{\mathrm{B}}$ de Johnson & 0,01327 & & $1^{\circ}$ \\
\hline \multirow{2}{*}{$\begin{array}{l}\hat{0} \\
\hat{0} \\
1 \\
0\end{array}$} & \multirow[b]{2}{*}{675} & Normal & 0,04980 & \multirow[b]{2}{*}{0,05235} & $1^{\circ}$ \\
\hline & & $\mathrm{S}_{\mathrm{B}}$ de Johnson & & & $2^{\circ}$ \\
\hline \multirow{2}{*}{$\begin{array}{l}\Rightarrow \\
= \\
=\end{array}$} & \multirow{2}{*}{328} & Normal & 0,02599 & \multirow{2}{*}{0,07509} & $1^{\circ}$ \\
\hline & & $\mathrm{S}_{\mathrm{B}}$ de Johnson & 0,03513 & & $2^{\circ}$ \\
\hline
\end{tabular}

Por meio dos resultados do teste de KolmogorovSmirnov, pode-se observar que quanto maior o número de dados utilizados para proceder à distribuição de frequências, menor é o valor de $D_{t a b}$, dificultando a existência de aderência satisfatória da fdp (Tabela 3 ).

A fdp Normal apresentou melhor ajuste para quatro classes de idade (5 a 5,9; 6 a 6,9; 10 a 10,9 e 11 a 11,9 anos), o que converge com resultados encontrados 
por Carelli Netto (2008) (Tabela 3). Para determinar a distribuição diamétrica de povoamentos de Pinus taeda em diferentes espaçamentos e idades, Carelli Netto (2008) ajustou as fdp's de Weibull, Normal, Log-Normal e Gamma. Utilizando os testes de aderência, entre eles o de Kolmogorov-Smirnov, tendo verificado que a função Normal apresentou melhor ajuste para as idades de 6,8 e 10 anos, tanto para o espaçamento de $2 \mathrm{~m}$ x $2 \mathrm{~m}$ como para o de $2 \mathrm{~m} \mathrm{x} 3 \mathrm{~m}$.

A função de densidade probabilística $\mathrm{S}_{\mathrm{B}}$ de Johnson apresentou melhor ajuste para três classes de idade (7 a 7,9; 8 a 8,9, e 9 a 9,9 anos), de acordo com a Tabela 3. Resultados semelhantes foram obtidos por Bartoszeck et al. (2004), quando ajustaram as funções de densidade probabilísticas Normal, Log-Normal, Gama, Beta, Weibull 2 e 3 parâmetros e $S_{B}$ de Johnson para descrever as distribuições diamétricas de povoamentos de bracatinga (Mimosa scabrella Bentham), na região metropolitana de Curitiba, PR. Por meio do teste de Kolmogorov-Smirnov, os autores concluíram que a função $S_{B}$ de Johnson foi a que apresentou melhor desempenho.

As distribuições Normal e $S_{B}$ de Johnson também apresentaram os melhores desempenhos em estudos realizados por Machado et al. (2009a), para avaliar o comportamento de funções de densidade probabilística no ajuste da distribuição diamétrica de Araucaria angustifolia, em Curitiba, PR.

Finger (1982) estudou a distribuição dos diâmetros de povoamentos de Acacia mearnsii, localizados em Montenegro, RS. Por meio dos testes KomogorovSmirnov e Logarítmo da Probabilidade, tendo constatado que a função $S_{B}$ de Johnson foi a melhor fdp para descrever a distribuição de diâmetros em todas as idades estudadas (3,5 a 7,5 anos).

\section{Dinâmica da distribuição diamétrica}

De acordo com a posição da Tabela 3, a melhor fdp para avaliar a evolução da distribuição diamétrica ao longo do tempo foi a fdp Normal. Além disso, optamos pela Normal por sua simplicidade. Machado et al. (2009a), estudando distribuição diamétrica de Araucaria angustifolia em um fragmento de Floresta Ombrófila Mista, também utilizaram uma posição e encontraram a fdp Normal como a que melhor representou a distribuição diamétrica.

$\mathrm{Na}$ Figura 1 encontram-se as frequências observadas e as frequências estimadas (Função Normal), em número de árvores por hectare, para distribuição diamétrica de povoamentos de Pinus taeda, da região de Caçador, SC.

Os povoamentos de Pinus taeda da região de Caçador, $\mathrm{SC}$, apresentaram distribuição diamétrica unimodal em todas as classes de idade (Figuras 1 e 2).

Nas primeiras idades, o número de árvores por hectare foi maior nas menores classes de diâmetro. Com o passar do tempo, o número de árvores por hectare diminuiu nas menores classes diamétricas e aumentou nas maiores, devido ao crescimento das árvores, o que fez com que as curvas de distribuição diamétrica se deslocassem para a direita (Figuras 1 e 2). O deslocamento do pico da distribuição diamétrica para a direita indicou o aumento do valor da moda dos diâmetros com a idade. Esse deslocamento das curvas de distribuição dos diâmetros também foi encontrado por Machado et al. (2006), estudando a dinâmica da distribuição diamétrica de povoamentos de bracatinga (Mimosa scabrella), na Região Metropolitana de Curitiba, PR, por Carelli Netto (2008), estudando Pinus taeda, em Otacílio Costa, SC, e por Leite et al. (2005), em um estudo sobre distribuição diamétrica de Eucalyptus sp., submetido a desbaste, na Bahia.

A Figura 2 ilustra a dinâmica da distribuição diamétrica dos povoamentos de Pinus taeda, da região de Caçador, SC, nas idades de 5 a 11,9 anos. 

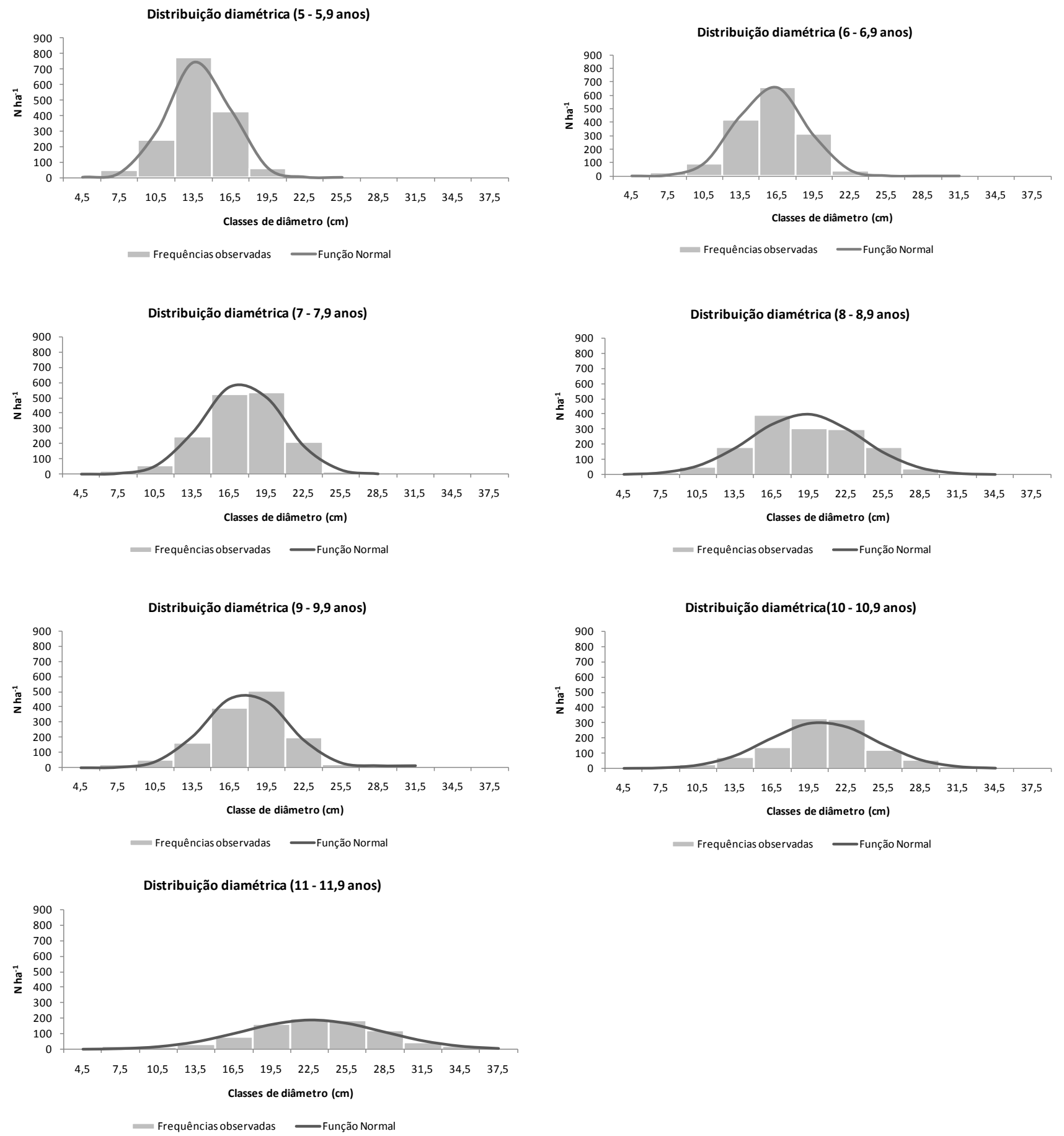

Figura 1. Frequências observadas e estimadas (função normal) para distribuição diamétrica de povoamentos de Pinus taeda L., da região de Caçador, SC, para as idades de 5 a 5,9 anos; 6 a 6,9; 7 a 7,9; 8 a 8,9; 9 a 9,9; 10 a 10,9 e 11 a 11,9 anos. 


\section{Função normal}

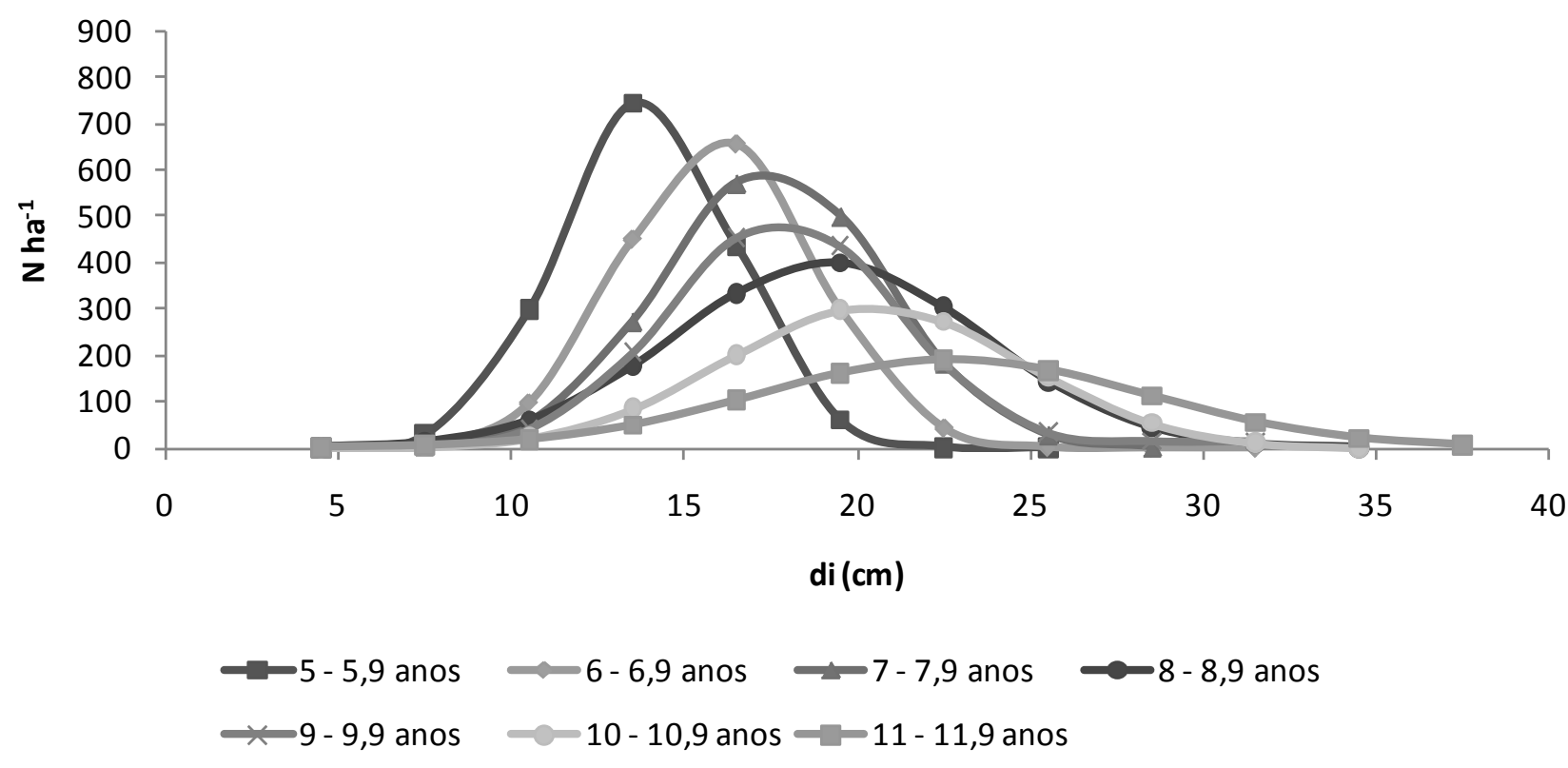

Figura 2. Distribuição diamétrica estimada (Função Normal) dos povoamentos de Pinus taeda L., da região de Caçador, SC, nas idades de 5 a 11,9 anos.

Ao comparar a distribuição diamétrica da menor para a maior classe de idade, verificou-se um "alargamento" das curvas de distribuição diamétrica decorrente do aumento na amplitude total dos diâmetros (Figura 2 e Tabela 1). O "alargamento" das curvas de distribuição dos diâmetros, com o passar dos anos, também foi constatado por Carelli Netto (2008), Leite et al. (2005), Machado et al. (2006) e Nogueira et al. (2006), trabalhando com diferentes espécies em diferentes regiões do Brasil.

Além disso, pode-se evidenciar que houve um "achatamento" nas curvas de distribuição diamétrica das menores para as maiores classes de idade (Figura 2). Esse "achatamento" aconteceu devido à redução do número de árvores ao longo dos anos, causada pelos desbastes e pela mortalidade das árvores.

O "achatamento" das curvas de distribuição dos diâmetros também foi encontrado por Leite et al. (2005), trabalhando com Eucalyptus sp., na Bahia, por Nogueira et al. (2006), em uma avaliação de um modelo de distribuição diamétrica ajustado para povoamentos de Tectona grandis L. f. submetidos a desbastes, no Mato Grosso, e por Machado et al. (2006), estudando bracatinga, no Paraná.

Em todas as classes de idade, exceto na classe de idade de 8 a 8,9 anos, a assimetria da distribuição diamétrica foi negativa ou à direita $\left(\gamma_{1}<0\right)($ Tabela 4 e Figuras 1 e 2). A idade de 8 a 8,9 anos apresentou uma assimetria da distribuição diamétrica mais próxima a zero, o que sugere que ela se aproxima de uma distribuição perfeitamente simétrica (Figura 1D). Machado et al. (2009a) também encontraram uma distribuição diamétrica simétrica ( $\gamma_{1} \cong 0$ ) para povoamentos de Araucaria angustifolia, em Curitiba, PR.

Tabela 4. Parâmetros de assimetria e curtose da distribuição diamétrica dos povoamentos de Pinus taeda L., da região de Caçador, SC.

\begin{tabular}{ccc}
\hline \multirow{2}{*}{$\begin{array}{l}\text { Idade } \\
(\text { anos })\end{array}$} & \multicolumn{2}{c}{ Parâmetros } \\
\cline { 2 - 3 } $5-5,9$ & $-0,39728$ & Curtose $\left(\mathrm{g}_{2}\right)$ \\
$6-6,9$ & $-0,46809$ & 0,77066 \\
$7-7,9$ & $-0,51901$ & 0,88086 \\
$8-8,9$ & 0,05064 & 0,71498 \\
$9-9,9$ & $-0,80480$ & $-0,09805$ \\
$10-10,9$ & $-0,12680$ & 1,21485 \\
$11-11,9$ & $-0,37521$ & 0,97402 \\
\hline
\end{tabular}

A curtose da distribuição diamétrica foi positiva em todas as idades, exceto na idade de 8 a 8,9 anos, a qual 
apresentou curtose negativa da distribuição diamétrica (Tabela 4). Assim, para as classes de idade de 5 a 5,9, 6 a $6,9,7$ a 7,9, 9 a 9,9, 10 a 10,9 e 11 a 11,9 anos, a curtose da distribuição diamétrica foi léptocúrtica, isto é, apresentou um cume mais pontiagudo quando comparado à curva Normal. Já para a idade de 8 a 8,9 anos, a curtose da distribuição diamétrica foi platicúrtica, ou seja, apresentou um cume menos pontiagudo que a curva Normal. A curtose da distribuição diamétrica também foi platicúrtica para povoamentos de Araucaria angustifolia no trabalho realizado por Machado et al. (2009a).

Resultados de assimetria negativa da distribuição diamétrica também foram encontrados por Machado et al. (2008), que estudaram o efeito da densidade e do sítio sobre as curvas de distribuição diamétrica, em povoamentos de bracatinga (Mimosa scabrella), em Curitiba, PR. Para o sítio I, esses pesquisadores encontraram assimetria negativa nas idades de 4,1 e 5 anos. Já para o sítio II, encontraram assimetria negativa para as idades 4,2; 5,4; 6,3 e 7,6 anos. Convém ressaltar que o sítio I apresenta melhores condições para o desenvolvimento e crescimento das árvores, quando comparado com as condições de crescimento e desenvolvimento proporcionadas pelo sítio II. Quanto à curtose, Machado et al. (2008) observaram que ocorreu um "achatamento" das curvas de distribuição diamétrica da maior para a menor densidade e do sítio menos produtivo para o mais produtivo.

Carelli Netto (2008) encontrou assimetria positiva e um leve "achatamento" das curvas em relação ao eixo horizontal, para povoamentos de Pinus taeda com idades de 6 a 18 anos.

Finger (1982), estudando a distribuição de diâmetros em acácia negra (Acacia mearnsii) em diferentes povoamentos e idades, constatou que na idade de 3,5 anos a distribuição diamétrica foi assimétrica positiva e a curtose foi platicúrtica. Para as idades de 4,5; 5,5; 6,5 e 7,5 anos, a distribuição dos diâmetros foi assimétrica negativa e a curtose foi platicúrtica.

Machado et al. (2010), estudando a distribuição da altura total, da área transversal e do volume individual de Araucaria angustifolia em Curitiba, PR, encontraram assimetria positiva para área transversal e volume individual. Já para altura, as curvas foram simétricas $\left(\gamma_{1} \cong 0\right)$ e a curtose foi mesocúrtica. Machado et al. (2009b) também encontraram assimetria positiva, ao analisar curvas de distribuição diamétrica de povoamentos de cedro e cambará, localizados em Curitiba, PR.

As distribuições diamétricas de todas as idades dos diferentes povoamentos, quando representadas em um mesmo gráfico, resultaram em uma distribuição diamétrica decrescente, também chamada de exponencial negativa ou J-invertido (Figura 2).

O padrão de distribuição decrescente ocorreu devido à redução do número de árvores por hectare e ao aumento da amplitude diamétrica ao longo dos anos, resultando, respectivamente, em um "achatamento" e um "alargamento" das curvas de distribuições diamétricas com o passar dos anos. O crescimento em diâmetro fez com que as curvas de distribuição dos diâmetros se deslocassem para a direita.

Esse comportamento de distribuição diamétrica decrescente, quando representada para povoamentos de várias idades, também foi encontrado por Nogueira et al. (2006), que estudou Tectona grandis, por Leite et al. (2005), estudando Eucalyptus sp., por Arce (2004), em uma pesquisa sobre a modelagem da estrutura de florestas clonais de Populus deltoides Marsh, e por Carelli Netto (2008), que estudou a dinâmica da distribuição diamétrica de povoamentos de Pinus taeda de 6 a 18 anos, em diferentes espaçamentos.

O padrão de distribuição decrescente, apresentado pelas distribuições diamétricas de todas as idades, apresentadas em conjunto, não favorece o manejo florestal dos povoamentos, como se faz em uma floresta multiânea, com o conceito de floresta balanceada. Esse padrão de distribuição dos diâmetros tem a finalidade, simplesmente, de analisar a dinâmica da distribuição diamétrica com o passar do tempo. O manejo florestal nos povoamentos é realizado tentando-se buscar sempre a otimização por povoamento florestal, dentro de cada idade, e não considerando todos os povoamentos de forma conjunta.

\section{Conclusões}

A distribuição diamétrica de cada classe de idade dos povoamentos de Pinus taeda, da região de Caçador, SC, é unimodal. No entanto, ao representar a distribuição diamétrica de todas as idades em um mesmo gráfico, é obtida uma distribuição diamétrica decrescente, também chamada de exponencial negativa ou J-invertido.

Para as idades de 5 a 7,9 anos, a fdp Normal e a $\mathrm{S}_{\mathrm{B}}$ de Johnson não apresentam aderência satisfatória. 
A fdp $\mathrm{S}_{\mathrm{B}}$ de Johnson e a Normal apresentam melhor aderência para as idades de 8 a 9,9 e 10 a 11,9 anos, respectivamente.

O teste de Kolmogorov-Smirnov mostra-se sensível à medida que se aumenta o número de diâmetros utilizados para o ajuste da fdp, sendo que quanto maior este valor, menor é a chance do teste acusar boa aderência para a fdp ajustada.

Para estudar a dinâmica da distribuição diamétrica de Pinus taeda, na região de Caçador, SC, optou-se pela fdp Normal, devido ao teste de Kolmogorov-Smirnov e à simplicidade desta fdp.

\section{Referências}

ALVAREZ GONZALEZ, J. G.; RUIZ GONZALEZ, A. D. Analisis y modelizacion de las distribuciones diametricas de Pinus pinaster AIT. em Galicia. Investigacion Agraria, Sistemas y Recursos Forestales, Lugo, v. 7, p. 123-137, 1998.

ARCE, J. E. Modelagem da estrutura de florestas clonais de Populus deltoides Marsh. através de distribuições diamétricas probabilísticas. Ciência Florestal, Santa Maria, RS, v. 14, n. 1, p. 149-164, 2004.

BARTOSZECK, A. C. de P. e S.; MACHADO, S. do A.; FIGUEIREDO FILHO, A.; OLIVEIRA, E. B. A distribuição diamétrica para bracatingais em diferentes idades, sítios e densidades na região metropolitana de Curitiba. Floresta, Curitiba, v. 34, n. 3, p. 305-323, 2004.

CARELLI NETTO, C. Dinâmica da distribuição diamétrica de povoamentos de Pinus taeda $\mathrm{L}$. em diferentes idades e espaçamentos. 2008. 106 f. Dissertação (Mestrado em Engenharia Florestal) - Universidade Federal de Santa Maria, Santa Maria, RS.

CIRAM. Mapa de solos: unidade de planejamento regional meio oeste catarinense UPR 2. In: EPAGRI. Estudos básicos regionais de Santa Catarina. Florianópolis: EPAGRI, 2002. 1 CD.

CLUTTER, J. L.; FORTSON, J. C.; PIENNAR, L. V.; BRISTER, G. H.; BAILEY, R. L. Timber management: a quantitative approach. Nova York: John Wiley \& Sons, 1983. 333 p.

EPAGRI. Zoneamento agroecológico e socioeconômico do estado de Santa Catarina. [Florianópolis, 2010]. Disponível em: $<$ http://ciram.epagri.sc.gov.br/ciram_arquivos/arquivos/portal/ agricultura/zoneAgroecologico/ZonAgroeco.pdf > . Acesso em: 20 abr. 2010.

FINGER, C. A. G. Distribuição de diâmetros em Acácia Negra (Acacia mearnsii de Wild), em diferentes povoamentos e idades. 1982. 146 f. Dissertação (Mestrado em Ciências Florestais) - Universidade Federal do Paraná, Curitiba.

LEITE, H. G.; NOGUEIRA, G. S.; CAMPOS, J. C. C.; SOUZA, A. L.; CARVALHO, A. Avaliação de um modelo de distribuição diamétrica ajustado para povoamento de Eucalyptus sp. submetidos a desbaste. Revista Árvore, Viçosa, MG, v. 29, n. 2, p. 271-280, 2005 .
DE LIOCOURT, F. De l'amenagement des sapinières. Bulletin trimestriel, Besançon, p. 396-409, juil. 1898.

LOETSCH, F.; ZÖHRER, F.; HALLER, K. E. Forest Inventory: volume II. inventory data collected by terrestrial measurements and observations, data processing in forest inventory... München: BLV Verlagsgesellschaft, 1973. 469 p.

MACHADO, S. do A.; AUGUSTYNCZIK, A. L. D.; NASCIMENTO, R. G. M.; FIGURA, M. A.; SILVA, L. C. R. da; MIGUEL, E. P.; TÉO, S. J. Distribuição diamétrica de Araucária angustifólia (Bert.) O. Ktze. em um fragmento de Floresta Ombrófila Mista. Scientia Agraria, Curitiba, v. 10, n. 2, p. 103110, 2009a.

MACHADO, S. do A.; AUGUSTYNCZIK, A. L. D.; NASCIMENTO, R. G. M.; TÉO, S. J; MIGUEL, E. P.; FIGURA, M. A.; SILVA, L. C. R. da. Funções de distribuição diamétrica em um fragmento de Floresta Ombrófila Mista. Ciência Rural, Santa Maria, RS, v. 39, n. 8, p. 2428-2434, 2009b.

MACHADO, S. do A.; BARTOSZECK, A. C. de P. e S.; FIGUEIREDO FILHO, A.; OLIVEIRA, E. B. de. Dinâmica da distribuição diamétrica de Bracatingais na região metropolitana de Curitiba. Revista Árvore, Viçosa, MG, v. 30, n. 5, p. 759-768, 2006.

MACHADO, S. do A.; BARTOSZECK, A. C. de P. e S.; FIGUEIREDO FILHO, A.; OLIVEIRA, E. B. de. Efeito da densidade e do sítio sobre as curvas de distribuição diamétrica em Bracatingais Nativos (Mimosa scabrella benth.) da região metropolitana de Curitiba. Ambiência, Guarapuava, v. 4, n. 1, p. 37-50, 2008.

MACHADO, S. do A.; NASCIMENTO, R. G. M.; MIGUEL, E. P.; TÉO, S. J.; AUGUSTYNCZIK, A. L. D. Distribution of total height, transverse area and individual volume for Araucaria angustifolia (Bert.) O. Kuntze. Cerne, Lavras, MG, v. 16, n. 1, p. 12-21, 2010.

MEYER, H. A. Structure, growth, and drain in balanced uneven-aged forests. Journal of Forestry, Bethesda, v. 50, n. 2, p. 85-92, 1952.

NOGUEIRA, S. G.; LEITE, H. G.; CAMPOS, J. C. C.; TAKIZAWA, F. H. T.; COUTO, L. Avaliação de um modelo de distribuição diamétrica ajustado para povoamentos de Tectona grandis submetidos à desbaste. Revista Árvore, Viçosa, MG, v. 30, n. 3, p. 377-387, 2006.

SCHAAF, L. B.; FIGUEIREDO FILHO, A.; GALVÃO, F.; SANQUETTA, C. R. Alteração da estrutura diamétrica de uma Floresta Ombrófila Mista no período entre 1979 e 2000. Revista Árvore, Viçosa, MG, v. 30, n. 2, p. 283-295, 2006.

SCHNEIDER, P. R.; FINGER, C. A. G.; BERNETT, L. G.; SCHNEIDER, P. S. P; FLEIG, F. D. Estimativa dos parâmetros da função de densidade probabilística de Weibull por regressão aninhada em povoamento desbastado de Pinus taeda L. Ciência Florestal, Santa Maria, RS, v. 18, n. 3, p. 381-392, 2008.

SCOLFORO, J. R. S. Biometria florestal: modelos de crescimento e produção florestal. Lavras, MG: UFLA/FAEPE, 2006. 393 p.

SCOLFORO, J. R. S. Modelagem do crescimento e da produção de florestas plantadas e nativas. Lavras, MG: UFLA/FAEPE, 1998. $452 \mathrm{p}$. 